\title{
A sex-role-preference model for HIV transmission among men who have sex with men in China
}

\author{
Jie Lou1 ${ }^{1}$, Jianhong $\mathrm{Wu}^{2}$, Li Chen ${ }^{1}$, Yuhua Ruan ${ }^{3}$ and Yiming Shao*3
}

Address: ${ }^{1}$ Department of Mathematics, Shanghai University, 99 Shangda Road Shanghai, 200444, PR China, ${ }^{2}$ MITACS Centre for Disease Modeling, York University, Toronto, Ontario, Canada M3J 1P3 and ${ }^{3}$ Department of Research on Virology and Immunology, National Center for AIDS/STD Control and Prevention, 27 Nanwei Road, Beijing, 100050, PR China

Email: Jie Lou - jie.lou@staff.shu.edu.cn; Jianhong Wu - wujh@yorku.ca; Li Chen - chenli07720183@shu.edu.cn;

Yuhua Ruan - ruanyuhua92@gmail.com; Yiming Shao* - yshao@bbn.cn

* Corresponding author

Published: 18 November 2009

BMC Public Health 2009, 9(SuppI I):SI0 doi:I0.II86/I47I-2458-9-SI-SI0

This article is available from: http://www.biomedcentral.com/I47I-2458/9/SI/SIO

(C) 2009 Lou et al; licensee BioMed Central Ltd.

This is an open access article distributed under the terms of the Creative Commons Attribution License (http://creativecommons.org/licenses/by/2.0), which permits unrestricted use, distribution, and reproduction in any medium, provided the original work is properly cited.

\begin{abstract}
Background: Men who have sex with men (MSM) are much more likely to be infected with HIV than the general population. China has a sizable population of MSM, including gay, bisexual men, money boys and some rural workers. So reducing HIV infection in this population is an important component of the national HIV/AIDS prevention and control program.
\end{abstract}

Methods: We develop a mathematical model using a sex-role-preference framework to predict HIV infection in the MSM population and to evaluate different intervention strategies.

Results: An analytic formula for the basic reproduction ratio $R_{0}$ was obtained; this yields $R_{0}=$ 3.9296 in the current situation, so HIV will spread very fast in the MSM population if no intervention measure is implemented in a timely fashion. The persistence of HIV infection and the existence of disease equilibrium (or equilibria) are also shown. We utilized our model to simulate possible outcomes of antiretroviral therapy and vaccination for the MSM population. We compared the effects of these intervention measures under different assumptions about MSM behaviour. We also found that $R_{0}$ is a decreasing function of the death rate of HIV-infected individuals, following a power law at least asymptotically.

Conclusion: HIV will spread very fast in the MSM population unless intervention measures are implemented urgently. Antiretroviral therapy can have substantial impact on the reduction of HIV among the MSM population, even if disinhibition is considered. The effect of protected sexual behaviour on controlling the epidemic in the MSM population largely depends on the sex-ratio preference of different sub-populations.

\section{Background}

The report from the American Foundation for AIDS Research [1] suggests that the group originally at the most risk of HIV - gay and bisexual men - still remains at the highest risk. This is largely due to anal sex which, when unprotected, carries a high risk of HIV transmission, espe- cially for the receptive partner. Men who have sex with men (MSM) are 19 times more likely to be infected with HIV than the general population. Gay and bisexual men are only a part of the total MSM population, since MSM is a description of a behavioural phenomenon, not an identity. 
China's first, and most recent, official figure on male homosexuality was released in 2004, putting the total of gay men in the country at between five and ten million [2]. But this is only a conservative estimation [3]. The HIV infection rate among gay men in China is climbing at an alarming rate, largely due to neglecting this subpopulation. Recent studies suggest unprotected risk behaviour or sexually transmitted diseases (STDs) among MSM have been found in several cities in China. Disturbing HIV prevalence rates from 1.0 to 5.0\% among MSM have been reported in several urban cities [4]; higher than the overall prevalence $(0.05 \%)$ for China. Without timely action, MSM could become the second most risky group for HIV infection following injection drug users in China.

In China, sociologists and public health workers have long been aware of the commercial sex workers serving MSM, who are called money boys. Beijing, for example, has thousands of male sex workers, working in bathhouses, bars and clubs or finding their own clients on the streets or via the internet $[5,6]$. It is shown in [5] that, even if money boys are normally managed by a so-called "Mommy", it is not uncommon for some of them to suffer physical violence and rape from clients [7]. In such circumstances, it is hardly realistic to hope that male sex workers will always use condoms. Migrant rural workers now also become a source of MSM [5,6]. China's fastgrowing economy creates a lot of new jobs for migrant rural workers, who move frequently between big cities and their home town. A sizable proportion of these migrant workers have sex with men. Some of them also act as money boys to some old or not so popular gay men; in this situation, migrant workers normally only prefer insertive anal intercourse (AI) [6]. Thus, the population of money boys includes both professional money boys, and also a small number of rural workers. Some deterministic models have been proposed to understand the HIV epidemic in homosexual populations [8,9]. In [8], Valle et al looked at the impact of education, temporarily effective vaccines and therapies on the dynamics of HIV in homosexually active populations. Their study assumed that some individuals possess one or two mutant alleles (like D32 of CCR5) that prevent the successful invasion or replication of HIV, and the study examined separate or combined effects of therapies, education, vaccines and genetic resistance. Breban et al. [9] evaluated the potential impact of rectal microbicides for reducing HIV transmission in bathhouses. In addition, Tan \& Kiang [10] proposed a state space model (Kalman filter model) for the HIV epidemic in homosexual populations stratified into sub-populations by their sexual activity levels.

None of the aforementioned studies seems to have considered the sex-role preference, which is quite important to address the HIV spread in some MSM populations. In [11], Yee gave a summary of a study that tested a more elaborate categorization about the correlations between sex-role preference and physical preferences for partners among gay men. According to the sex roles of gay men, as Top or Bottom - preference for insertive AI and preference for receptive AI respectively - the study found that sex-role preference is indeed correlated with differences in physical preferences for a sexual partner among gay men. The categorization tested in this study includes 6 categories: Only Bottom; Versatile, but prefer Bottom; Versatile, equal; Versatile, but prefer Top; Only Top; Never had anal

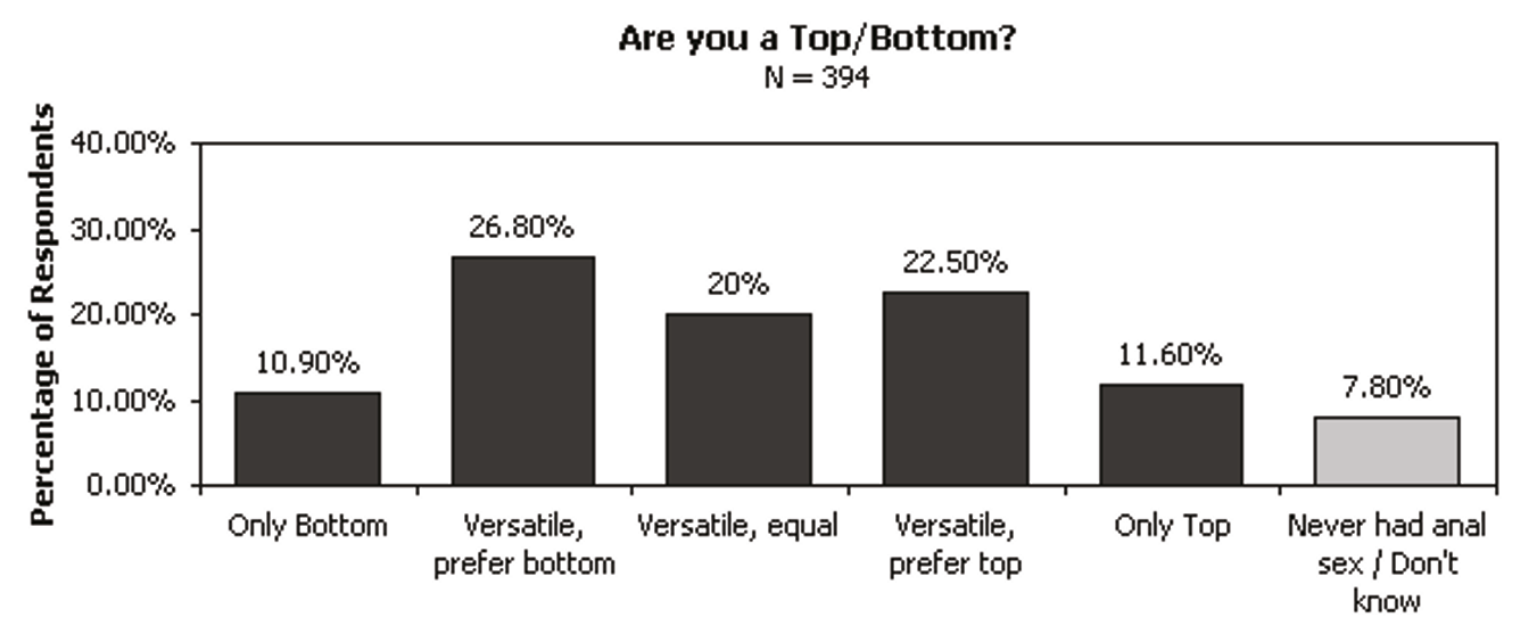

Figure I

Distribution of sex roles of MSM. Sex roles should be thought of as a continuous spectrum that maps onto a continuous spectrum of physical preferences [II]. 
sex/Don't Know. All respondents in the study were volunteers recruited from http://www.gay.com chatrooms in January 2002. They were asked to participate in an online questionnaire. A total of 396 respondents completed the survey. Figure 1 gives the distribution of how respondents categorized themselves into the elaborate sex role categories above. The data of [11] suggests that sex roles should be thought of as a continuous spectrum that maps onto a continuous spectrum of physical preferences.

In our study here, we assume that HIV transmission takes place exclusively through $\mathrm{AI}$ (both receptive and insertive acts occur). We develop a mathematical model, based on the above categorization and the assumption that the viral transmission probability per anal sex act is different when transmission happens through receptive acts or insertive acts. Therefore, we divide MSM into three subgroups:

- Only Bottom, including gay and bisexual men who prefer receptive $\mathrm{AI}$, and some money boys;

- Versatile, including all gay and bisexual men who are versatile in sex role, and some migrant rural workers;

- Only Top, including gay and bisexual men who prefer insertive AI, and some money boys (such as some migrant rural workers who earn subsidy income by having sex with gay men).

We use this model to examine the effect of highly active antiretroviral therapy on controlling the HIV spread in the MSM population. HAART has led to dramatic decrease in morbidity and mortality among individuals infected with HIV. However, HAART coverage remains suboptimal, even in the resource-rich areas of the world. Our modelbased simulations therefore assume a small portion of MSM in China with HIV-1 will start to take HAART. Since HAART predictably decreases plasma HIV-1 RNA levels to below the levels of detection of currently available assays [12], we assume that individuals taking HAART are no longer infectious. An increase in adverse behaviour can result from the availability of interventions, the so-called disinhibition. Several early mathematical modeling studies raised the concern that any possible benefit of HAART on the spread of HIV could be readily offset by even modest increases in HIV risk behaviour [13]. However, our model shows that antiretroviral therapy for MSM in China will have both individual and public-health benefits even if risk behaviour increases. We also discuss the effect of vaccination for general MSM in order to compare different strategies.

\section{Methods}

Mathematical model

We develop an ordinary differential equation (ODE) model describing HIV infection among the MSM population according to individual's sex-role preference. We use $S_{T^{\prime}} S_{B^{\prime}} S_{V}$ to represent the susceptive MSM in the Only Top, the Only Bottom, and the Versatile category respectively. We use $I_{T}$ to represent the infected MSM in the Only Top category; $I_{B}$ and $I_{V}$ are defined similarly. Then we have the following ODE model:

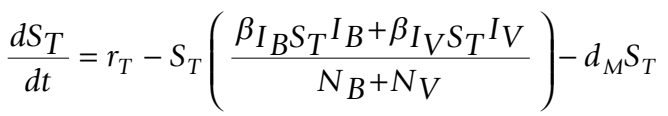

$$
\begin{aligned}
& \frac{d I_{T}}{d t}=S_{T}\left(\frac{\beta_{I_{B} S_{T} I_{B}+\beta_{I_{V}} S_{T} I_{V}}}{N_{B}+N_{V}}\right)-d_{I} I_{T} \\
& \frac{d S_{V}}{d t}=r_{V}-S_{V}\left(\frac{\beta_{I_{T}} S_{V} I_{T}+\beta_{I_{B}} S_{V} I_{B}+\beta_{I_{V}} S_{V} I_{V}}{N_{T}+N_{V}+N_{B}}\right)-d_{M} S_{V} \\
& \frac{d I_{V}}{d t}=S_{V}\left(\frac{\beta_{I_{T}} S_{V} I_{T}+\beta_{I_{B}} S_{V} I_{B}+\beta_{I_{V}} S_{V} I_{V}}{N_{T}+N_{V}+N_{B}}\right)-d_{I} I_{V} \\
& \frac{d S_{B}}{d t}=r_{B}-S_{B}\left(\frac{\beta_{I_{T}} S_{B} I_{T}+\beta_{I} S_{B} I_{V}}{N_{T}+N_{V}}\right)-d_{M} S_{B} \\
& \frac{d I_{B}}{d t}=S_{B}\left(\frac{\beta_{I_{T}} S_{B} I_{T}+\beta_{I_{V}} S_{B} I_{V}}{N_{T}+N_{V}}\right)-d_{I} I_{B}
\end{aligned}
$$

where $N_{T}=S_{T}+I_{T}, N_{V}=S_{V}+I_{V}$ and $N_{B}=S_{B}+I_{B}$ denote the total population of the Only Top, the Versatile and the Only Bottom categories, respectively. Note that the Only Top category can have sex with the Only Bottom and the Versatile population. The Only Bottom category can have sex with the Only Top and the Versatile population. The Versatile category can have sex with all categories. In this model, we assume that susceptible and infected MSM can die at rates $d_{M}$ and $d_{I}$ respectively. Also, we assume new MSM are recruited into the appropriate susceptible compartment at rates $r_{T}, r_{B}$ and $r_{V}$ respectively.

\section{The HIV transmission rate $\left(\beta_{\mathrm{yx}}\right)$}

The key to quantify the transmission of HIV is the parameter $\beta_{\gamma x}$, the transmission rate that an individual from compartment $y$ infects his partners from compartment $x$. We shall use the following formula to estimate the HIV transmission rate through either insertive or receptive AI:

$$
\beta_{y x}=n_{x} c_{x} h_{y x}\left(1-\eta^{c} \rho^{c}\right)\left(1-\alpha_{y}\left(1-\rho^{c}\right) v_{y} \eta^{c}\right)\left(1+\mu^{s} \psi^{s}\right) .
$$

The HIV transmission rate through anal sex in MSM depends on six quantities:

- the number of different AI sex partners per year, $n_{x^{\prime}}$ for individuals from compartment $x$; 
- the number of AI with each sex partner per year, $c_{x^{\prime}}$ for individuals from compartment $x$;

- the viral transmission probability per anal sex act, $h_{y x}$ i

- the level of protection against HIV infection due to condom usage (if condoms are used, HIV transmission is decreased by a factor of $\left(1-\eta^{c} \rho^{c}\right)$, where $\eta^{c}$ is the condom efficacy and $\rho^{c}$ is the proportion of condom use);

- the proportion of infected MSM who know that they are infected, $\alpha_{y}$. This term denotes the effect of the 2008 HIV census in MSM population, where many men discovered they were HIV positive; $v_{y}$ denotes the proportion of these infected MSM who begin to control their behaviour (such as condom use) to avoid the spreading of HIV, if they did not use condoms before they knew that they have been infected by HIV.
- other STIs increase both the rate of transmission and acquisition of HIV (the proportion with other STIs is assumed to be $\psi^{s}$, with $\mu^{s}$ being the multiplication factor for HIV);

\section{HAART and HIV vaccination in MSM}

We assume that only $20 \%$ of MSM with HIV- 1 start to take HAART each year in China, although some of the simulations below permit variable rates of HARRT treatment. To model the effects of HAART, we add one additional compartment to each of the infected groups. We assume that individuals taking HAART extend their lifespan by 5 years, so their annual death rate is 0.069 . Since HAART predictably decreases plasma HIV-1 RNA levels to below the level of detection of currently available assays [12], we also assume that individuals taking HAART are no longer infectious. This reduction of plasma HIV-1 leads to an increase in adverse behaviour (disinhibition), and we model this behaviour change by reducing condom use between MSM from $\rho^{c}$ to zero (again, some of the simulations below allow for variable condom use rates). We also consider the effect of a potential vaccine, by adding one

Table I: Parameters.

\begin{tabular}{|c|c|c|c|}
\hline Para & Description & Value & Source \\
\hline$r_{T}$ & source rate of Only-Top MSM & 96667 & estimate \\
\hline$r_{V}$ & source rate of Versatile MSM & 603950 & estimate \\
\hline$r_{B}$ & source rate of Only-Bottom MSM & 70817 & estimate \\
\hline$d_{M}$ & death rate of susceptible MSM & 0.022 & estimate \\
\hline$d_{1}$ & death rate of infected MSM & 0.105 & estimate \\
\hline$n_{T}$ & number of Al sex partners per year of Only-Top MSM & 11.5 & {$[16]$} \\
\hline$n_{V}$ & number of $\mathrm{Al}$ sex partners per year of Versatile MSM & 12.4 & {$[16]$} \\
\hline$n_{B}$ & number of Al sex partner per year of Only-Bottom MSM & 13.6 & [16] \\
\hline$c_{T}$ & number of $\mathrm{Al}$ acts with each sex partners per year of Only-Top MSM & 4.4 & {$[16]$} \\
\hline$c_{V}$ & number of $\mathrm{Al}$ acts with each sex partners per year of Versatile MSM & 4.5 & {$[16]$} \\
\hline$c_{B}$ & number of $\mathrm{Al}$ acts with each sex partners per year of Only-Bottom MSM & 4.2 & {$[16]$} \\
\hline$h_{T B}$ & transmissibility of HIV from Top to Bottom & 0.01 & {$[9,20]$} \\
\hline$h_{V B}$ & transmissibility of HIV from Versatile to Bottom & 0.01 & {$[9,20]$} \\
\hline$h_{T V}$ & transmissibility of HIV from Top to Versatile & 0.01 & {$[9,20]$} \\
\hline$h_{B T}$ & transmissibility of HIV from Bottom to Top & 0.005 & {$[9,20]$} \\
\hline$h_{V T}$ & transmissibility of HIV from Versatile to Top & 0.005 & {$[9,20]$} \\
\hline$h_{B V}$ & transmissibility of HIV from Bottom to Versatile & 0.005 & {$[9,20]$} \\
\hline$h_{V V}$ & transmissibility of HIV from Versatile to Versatile & 0.0075 & estimate \\
\hline$\alpha_{y}$ & proportion of infected MSM who know they are infected & 0.15 & {$[16]$} \\
\hline$\eta^{c}$ & condom efficacy & 0.9 & {$[16]$} \\
\hline$\rho^{c}$ & rate of condom use & 0.3 & [16] \\
\hline$\psi^{s}$ & proportion with STI & 0.158 & [16] \\
\hline$\mu \mu^{s}$ & multiplication factor of STI for HIV & 2.89 & {$[21]$} \\
\hline$v_{y}$ & proportion of infected MSM who begin to control their behaviour & 0.5 & {$[16]$} \\
\hline
\end{tabular}

Note I: The mean values and standard errors of parameters $n_{T}, n_{V}, n_{B}, c_{T}, c_{V}$ and $c_{B}$ are

$$
\begin{gathered}
n_{T}=11.5 \pm 13.6, \quad n_{V}=12.4 \pm 12.2, \quad n_{B}=13.6 \pm 12.5 \\
c_{T}=4.4 \pm 4.2, \quad c_{V}=4.5 \pm 4.4, \quad c_{B}=4.2 \pm 4.5
\end{gathered}
$$

respectively.

Note 2: We estimate that the average life span of HIV infected individual is 9.5 years, so $d_{1}=0.105$. Also, we suppose young MSM begin homosexual sex behavior at 20 years and quit at 65 years, so $d_{M}=0.022$. 
compartment for each of the uninfected groups. This vaccine has the property that vaccinated individuals may become infected, if the efficacy of the vaccine is less than $100 \%$. In our baseline simulations, we assume that uninfected individuals are vaccinated at a rate of $20 \%$, (the same as the HAART rate), and we explore vaccine efficacies of $30 \%$ and $70 \%$. Equations for both the HAART model and the vaccination model can be found in Additional File 1.

\section{Parameters and initial values}

Table 1, Table 2 and Table 3 describe values of each of the biological and behavioural parameters, and Table 4 gives initial values. Some parameter values are calculated from the cohort study of China in 2008, which is on "high-risk behaviours and HIV/syphilis prevalence among men who have sex with men in Beijing". We also give the standard error for these parameter values. For example, the mean value of $n_{V}$ (the number of AI sex partner per year of Versatile MSM) is 12.4 and the standard error is 12.2. The mean value of $c_{V}$ (the number of AI with each sex partner per year of the Versatile) is 4.5 and the standard error is 4.4. The large variation in our original data - for example, the minimum value of $n_{V}$ is 1 but the maximum value is 120 - results in the large standard errors of the parameters.

For the initial values, we have the following. The cohort study in China in 2008 [16] estimated that the prevalence of HIV in MSM in China was about $1.5 \%$ by the end of 2008 [16]. The total population of China is about 1.3029 $\times 10^{9}$, and $50 \%$ of them are male. We suppose $3 \%$ of men in China have sex with men, $91.5 \%$ of whom experience AI, distributed as in Figure 1: 10.9\% are Only-Top, 69.3\% are Versatile and $11.6 \%$ are Only-Bottom. Then we have $1.8214 \times 10^{7} \mathrm{MSM}$ experiencing $\mathrm{AI}$, which is in the range estimated by [3]: the total number of gays in China is between $1.8 \times 10^{7}$ and $2.4 \times 10^{7}$. We also estimate that the number of professional money boys is about 10,000 . According to the newest report, the estimated number of rural workers was $2.2542 \times 10^{8}$ at the end of 2008 [14], $56 \%$ of whom are male. The study [5] estimated that about $10 \%$ of rural workers have homosexual sex behaviours to various extent, in which $20 \%$ experience AI. We

Table 2: Parameters and $\boldsymbol{R}_{\mathbf{0}}$.

\begin{tabular}{cccc}
\hline Parameters & Case I & Case 2 & Case 3 \\
\hline$h_{T B}, h_{V B}, h_{T V}$ & 0.01 & 0.001 & 0.001 \\
$h_{B T}, h_{V T}, h_{B V}$ & 0.005 & 0.0005 & 0.0005 \\
$h_{V V}$ & 0.0075 & 0.00075 & 0.00075 \\
$\rho^{c}$ & 0.3 & 0.6 & 0.6 \\
$d_{l}$ & 0.105 & 0.105 & 0.026 \\
\hline$R_{0}$ & 3.9296 & 0.2528 & 1.0212 \\
\hline
\end{tabular}

suppose that these AI-rural workers follow the distribution of $10.9 \%$ Only-Top ( $1 \%$ of them are money boys), $69.3 \%$ Versatile and $11.6 \%$ Only-Bottom. In summary, we estimate that the total number of MSM in China is about $2.0745 \times 10^{7}$. The initial values for each compartment can be found in Table 4 .

\section{Results and discussion \\ Model analysis: the basic reproductive ratio, $\mathbf{R}_{0}$}

Following the next-generation operator method of [15], we linearize the second, the fourth and the sixth equations of our model around the disease-free state and look for conditions that guarantee the growth of the three infected classes, $I_{T}, I_{V}$ and $I_{B}$.

For simplicity, we write $\beta_{V T}$ instead of $\beta_{I_{V} S_{T}}$. Other parameters labelled $\beta_{x y}$ have similar meanings. The Jacobian matrix of the second, the forth and the sixth equations of our model at the disease-free state can be rewritten in the following form:

$$
\frac{d X}{d t}=\left(F_{E_{0}}-V_{E_{0}}\right) X
$$

Where

$$
\begin{gathered}
X=\left[\begin{array}{lll}
I_{T}, & I_{V}, & I_{B}
\end{array}\right]^{T}, \\
F_{E_{0}}=\left[\begin{array}{ccc}
0 & \frac{r_{T} \beta_{V T}}{r_{B}+r_{V}} & \frac{r_{T} \beta_{B V}}{r_{B}+r_{V}} \\
\frac{r_{V} \beta_{T V}}{r_{T}+r_{V}+r_{B}} & \frac{r_{V} \beta_{V V}}{r_{T}+r_{V}+r_{B}} & \frac{r_{V} \beta_{B V}}{r_{T}+r_{V}+r_{B}} \\
\frac{r_{B} \beta_{T B}}{r_{T}+r_{V}} & \frac{r_{B} \beta_{V B}}{r_{T}+r_{V}} & 0
\end{array}\right]
\end{gathered}
$$

and

$$
V_{E_{0}}=\left[\begin{array}{ccc}
d_{I} & 0 & 0 \\
0 & d_{I} & 0 \\
0 & 0 & d_{I}
\end{array}\right] .
$$

Table 3: Infection rates of Table I.

\begin{tabular}{clc}
\hline Compartment & \multicolumn{1}{c}{ Description } & Value \\
\hline$\beta_{T B}$ & rate of infection by $I_{T}$ of $S_{B}$ & 0.5786 \\
$\beta_{T V}$ & rate of infection by $I_{T}$ of $S_{V}$ & 0.5653 \\
$\beta_{B T}$ & rate of infection by $I_{B}$ of $S_{T}$ & 0.2563 \\
$\beta_{V B}$ & rate of infection by $I_{V}$ of $S_{B}$ & 0.5786 \\
$\beta_{V T}$ & rate of infection by $I_{V}$ of $S_{T}$ & 0.2563 \\
$\beta_{B V}$ & rate of infection by $I_{B}$ of $S_{V}$ & 0.2826 \\
$\beta_{V V}$ & rate of infection by $I_{V}$ of $S_{V}$ & 0.4239
\end{tabular}


Table 4: Initial Conditions.

\begin{tabular}{ccc}
\hline Compartment & Description & Initial Value \\
\hline$S_{T}$ & susceptible Only-Top MSM & $2.4251 \times 10^{6}$ \\
$S_{V}$ & susceptible Versatile MSM & $1.5418 \times 10^{7}$ \\
$S_{B}$ & susceptible Only-Bottom MSM & $2.5908 \times 10^{6}$ \\
$I_{T}$ & infected Only-Top MSM & $3.6931 \times 10^{4}$ \\
$I_{V}$ & infected Versatile MSM & $2.3480 \times 10^{5}$ \\
$I_{B}$ & infected Only-Bottom MSM & $3.9455 \times 10^{4}$ \\
\hline
\end{tabular}

$R_{0}$ is the spectral radius of the next-generation matrix. Therefore, to find $R_{0}$ we must find the largest eigenvalue of $F_{E_{0}} V_{E_{0}}^{-1}$.

So

$$
R_{0}=\rho\left(F_{E_{0}} V_{E_{0}}^{-1}\right)
$$

that is

$R_{0}=\max _{|\lambda|}\left(\operatorname{det}\left[\begin{array}{ccc}\lambda & -\frac{r_{T} \beta_{V T}}{d_{I}\left(r_{B}+r_{V}\right)} & -\frac{r_{T} \beta_{B T}}{d_{I}\left(r_{B}+r_{V}\right)} \\ -\frac{r_{V} \beta_{T V}}{d_{I}\left(r_{T}+r_{V}+r_{B}\right)} & \lambda-\frac{r_{V} \beta_{V V}}{d_{I}\left(r_{T}+r_{V}+r_{B}\right)} & -\frac{r_{V} \beta_{B V}}{d_{I}\left(r_{T}+r_{V}+r_{B}\right)} \\ -\frac{r_{B} \beta_{T B}}{d_{I}\left(r_{T}+r_{V}\right)} & -\frac{r_{B} \beta_{V B}}{d_{I}\left(r_{T}+r_{V}\right)} & \lambda\end{array}\right]\right)$
Then the characteristic equation of $F_{E_{0}} V_{E_{0}}^{-1}$ is

$$
\lambda^{3}-d \lambda^{2}-(a c+b f+e g) \lambda+b d f-a e f-b c g=0,
$$

Where

$$
\begin{gathered}
a=\frac{r_{T} \beta_{V T}}{d_{I}\left(r_{B}+r_{V}\right)}, \quad b=\frac{r_{T} \beta_{B T}}{d_{I}\left(r_{B}+r_{V}\right)}, \quad c=\frac{r_{V} \beta_{T V}}{d_{I}\left(r_{T}+r_{V}+r_{B}\right)}, \quad d=\frac{r_{V} \beta_{V V}}{d_{I}\left(r_{T}+r_{V}+r_{B}\right)}, \\
e=\frac{r_{V} \beta_{B V}}{d_{I}\left(r_{T}+r_{V}+r_{B}\right)}, \quad f=\frac{r_{B} \beta_{T B}}{d_{I}\left(r_{T}+r_{V}\right)}, \quad g=\frac{r_{B} \beta_{V B}}{d_{I}\left(r_{T}+r_{V}\right)} .
\end{gathered}
$$

Let

$$
A=a c+b f+e g, \quad B=b d f-a e f-b c g, \quad C=\sqrt{81 B^{2}-12 A^{3}-3 A^{2} d^{2}-54 A B d-12 B d^{3}}
$$

and $D=\left(36 A d+8 d^{3}+12 C-108 B\right)^{\frac{1}{3}}$. Then

$$
R_{0}=\frac{d}{3}+\frac{D}{6}+\frac{2 A}{D}+\frac{2 d^{2}}{3 D}
$$

The reproductive number, $R_{0}$, is the number of secondary cases produced by a typical infected individual during his entire period of infectiousness in a demographically steady susceptible population. Calculating this number for our model is critical to determine whether HIV can

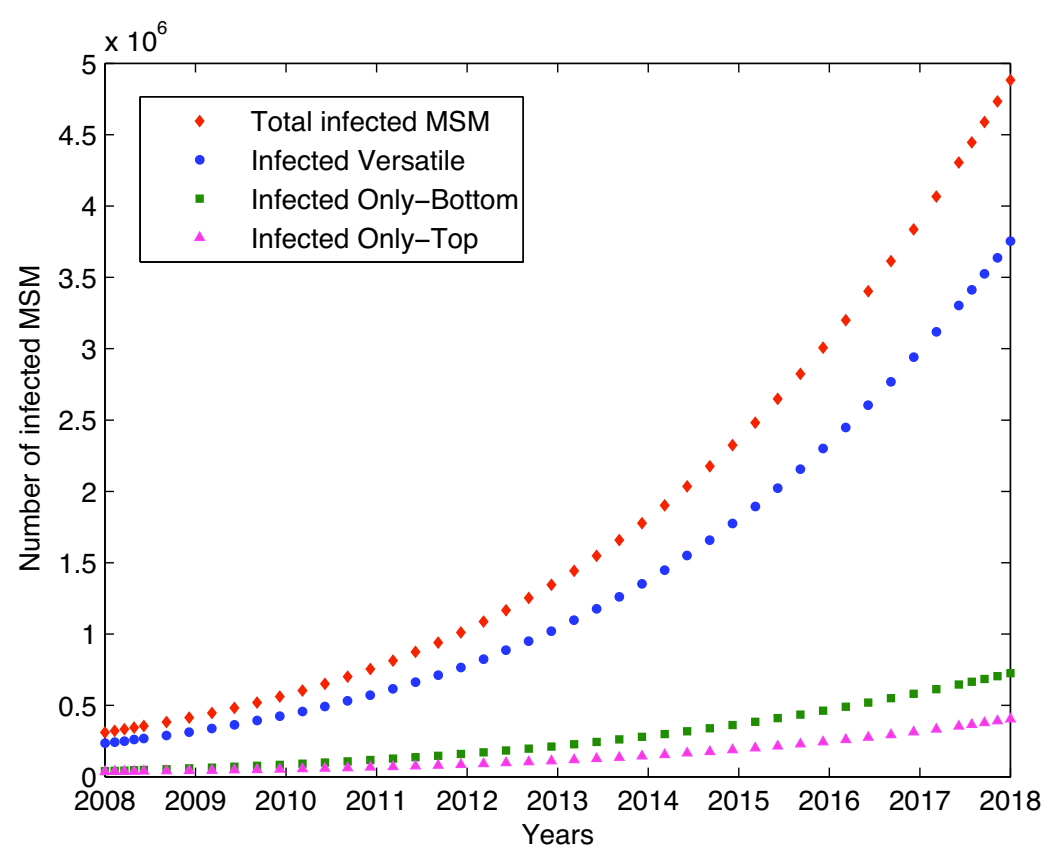

Figure 2

Dynamics of all sub-populations in the absence of intervention. Parameters used were as in Table I. Initial conditions were as in Table 4. With these parameters, we have $R_{0}=3.9296$ and hence the disease-free equilibrium is unstable and the disease is uniformly persistent. By the year 2018 the total number of newly infected MSM in China will be close to $4.88 \times 10^{6}$. That is, prevalence among MSM will reach over $21.7 \%$ by the year 2018 and the Versatile MSM alone will reach a prevalence rate of over $16.6 \%$. 
invade the MSM population and/or stabilize in the population. We can show that when $R_{0}<1$, HIV will not be sustained in this MSM population; otherwise, the infection will approach an endemic equilibrium of constant incidence and prevalence.

\section{Persistence of HIV infection}

By Thieme's persistence theory, we prove that the system is persistent of HIV infection when $R_{0}>1$; i.e., when $R_{0}>$ 1 , HIV will spread in the MSM population so long as one infected MSM is introduced in this population, regardless of whether he is an Only-Top, a Versatile or an Only-Bottom. We also get the existence of disease equilibrium (or equilibria) from the persistence of HIV infection. The proof is in Additional File 1.

\section{Numerical simulations}

\section{Outcomes without any intervention}

We start with the baseline analysis where we assume there is no intervention measure. Parameter values are chosen as in Table 1, estimated from the situation of 2008 in China [16]. We obtained that $R_{0}=3.9296$. In other words, one HIV-infected MSM can infect almost 4 other HIV-susceptible individuals in the same population before he dies. Since $R_{0}>1$, epidemic will be firmly established, and our simulation (Figure 2) predicts that, by the year 2018, the total number of newly infected MSM in China will be close to $4.88 \times 10^{6}$, and HIV prevalence among MSM will reach over $21.7 \%$. The infected Versatile MSM population will have a HIV prevalence rate of over $16.6 \%$. Note that the infected Versatile MSM increases much faster than the other two sub-populations (Figure 2). It shows the higher risk of this subpopulation if no intervention measure is implemented.

As far as the 10-year HIV prevalence prediction above is concerned, the outcomes are robust to most of the parameters in Table 1, except some special ones. Simulations show that the number of AI sex partners per year of Versatile MSM $\left(n_{V}\right)$ and the number of AI events with each sex partner per year for Versatile MSM $\left(c_{V}\right)$ are the two parameters which influence the final outcome most, and the condom use rate $\left(\rho^{c}\right)$ the least. Figure 3 shows the effect of different values of $n_{V}$ on the outcome of ten years' prevalence prediction, which shows the critical role played by the number of sex partners.

\section{Outcomes of HAART and vaccination}

The effects of HAART and vaccination are illustrated in Figure 4. Suppose the HAART rate in MSM is $20 \%$. If MSM population will not change their behaviour after receiving HAART (in our setting, this means that the condom using rate remains as $\rho^{c}=0.3$ ), then $R_{0}=1.3528$ and the prevalence can be greatly reduced (the solid-square curve). The empty-square curve shows the effect of HAART with behaviour change (disinhibition occurs, with the condom using rate $\left.\rho^{c}=0\right) . R_{0}=1.8138$ at this situation, which is still smaller than that without any intervention.

Suppose the vaccination rate in MSM is also 20\%. Then the effects of a potential vaccine with efficacy of $30 \%$ and $70 \%$ are shown as the solid-ring curve and the empty-ring curve, respectively. $R_{0}=2.8676$ and $R_{0}=1.4514$ for each situation. It shows that the effect of a potential vaccine

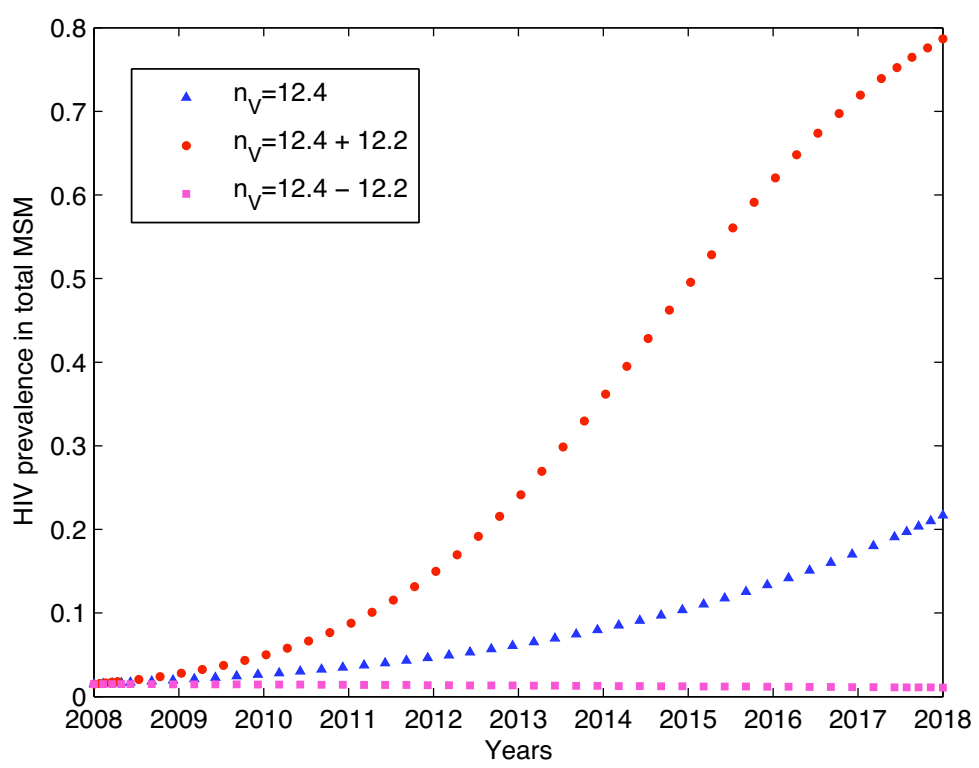

Figure 3

The outcome varies with errors in the parameter estimates. The effect of different $n_{V}$ (the number of Al sex partner per year of Versatile MSM) on the outcome of ten years' prevalence prediction. The mean value of $n_{V}=12.4$ and its standard error is 12.2. $R_{0}=7.1064$ for $n_{V}=24.6$, which is almost double of $R_{0}=3.9296$ of $n_{V}=12.4$. 


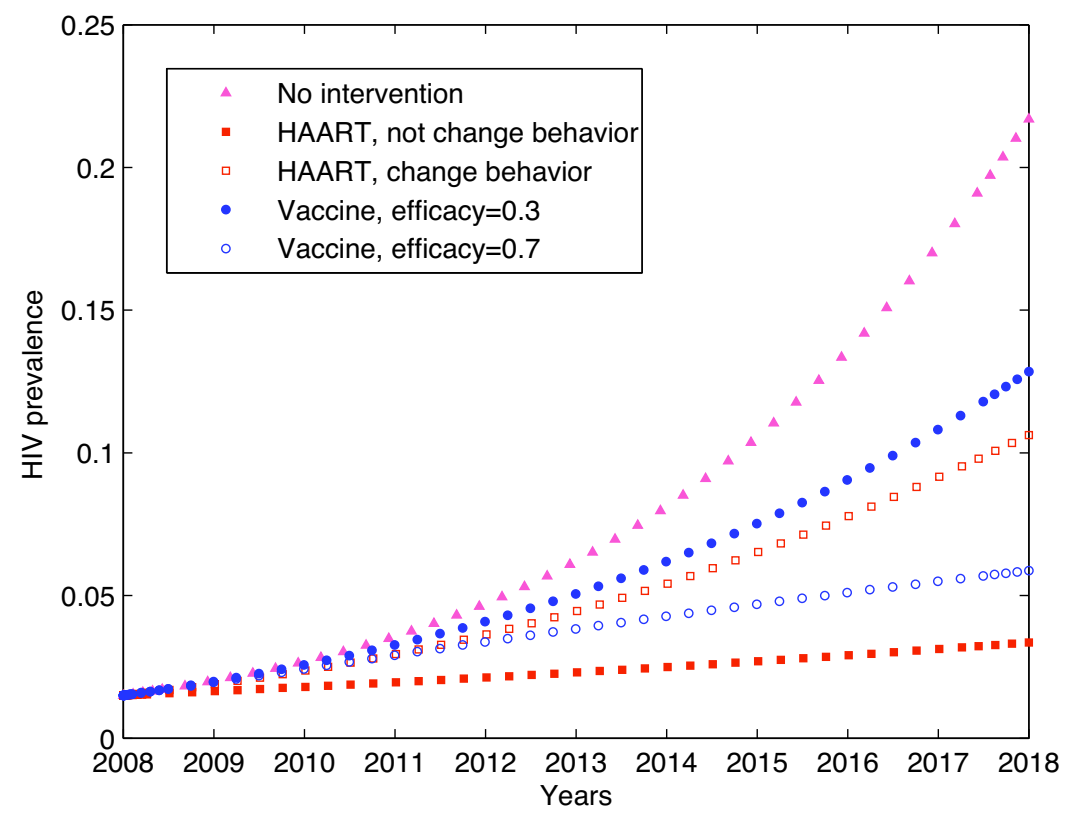

\section{Figure 4}

Comparison of dynamics for HIV-infected MSM. Comparison of dynamics for HIV infected MSM: no intervention, HAART and vaccination. All parameters used were the same as in Figure 2, except the condom use rate $\rho^{c}$ was either 0.30 or 0 . Additionally, for the HAART strategy, individuals taking HAART extend their lifespan by 5 years. So their death rate $d_{A}$ is 0.069 . For the vaccination strategy, uninfected individuals were vaccinated at a rate of $20 \%$, and the effect of the vaccine is $30 \%$ and $70 \%$ respectively. For these parameters, we have $R_{0}^{H A A R T}=1.3528$ for $\rho^{c}=0.30$ (i.e., MSM do not change behaviour), $R_{0}^{H A A R T}=1.8138$ for $\rho=0$ (i.e., MSM change behaviour), $R_{0}^{\text {vaccine }}=2.8676$ for $30 \%$ efficacy and $R_{0}^{\text {vaccine }}=1.45 \mathrm{I} 5$ for $70 \%$ efficacy. Whether MSM population change their behaviour or not after receiving HAART (in our setting, this means that the condom use rate remains at $\rho^{c}=0.30$ or decreases to zero), prevalence can be greatly reduced.

appears worse than that of HAART, even if the vaccine efficacy is as high as $70 \%$. Simulation shows that even if disinhibition occurs, the effect of HAART is still much better than that of no intervention. This result is remarkably different from those in [13], but agrees with those in [17].

Figure 5 shows the graph of $R_{0}$ as a function of the condom rate and the HAART rate, while Figure 6 plots a few contours of $R_{0}$. Figure 7 gives the graph of $R_{0}$ as a function of the vaccination rate, while Figure 8 plots a few contours of $R_{0}$. These illustrate the feasibility of controlling the HIV epidemic within the MSM population via a combination of condom use and HAART or a vaccination program.

Reproduction number and lifespan: power law

It was reported that certain medicines, especially some traditional Chinese medicines [18,19], which are reasonably cheap and easily available to HIV-infected individuals, can decrease the viral load and extend the lifespan of HIVinfected individuals. Here we design a simulation to simulate the impact of such medicines: we assume the trans- mission probability per anal sex is 10 times less than without the medicine, and the HIV-infected individuals have the reduced death rate $d_{I}=0.026$. Even under the assumption that MSM are more cautious in sexual behaviour (so that their condom using rate is doubled), we noticed that an endemic is maintained in the population since the basic production ratio $R_{0}=1.0212>1$ (Case 3 in Table 2, in comparison with those in Case 2). One of the reasons for this sustained infection is that the ratio $R_{0}$ is an decreasing function of the death rate $d_{I}$, following a power law at least asymptotically (See Figure 9).

\section{Conclusion}

We developed a mathematical model using a sex-rolepreference framework to predict HIV infection in the MSM population. An analytic expression of the basic reproduction ratio $R_{0}$ was obtained using model parameters, and we estimated the current $R_{0}$ as 3.9296 .

Our simulations suggest that both antiretroviral therapy and a potential vacce are powerful interventions, even if 


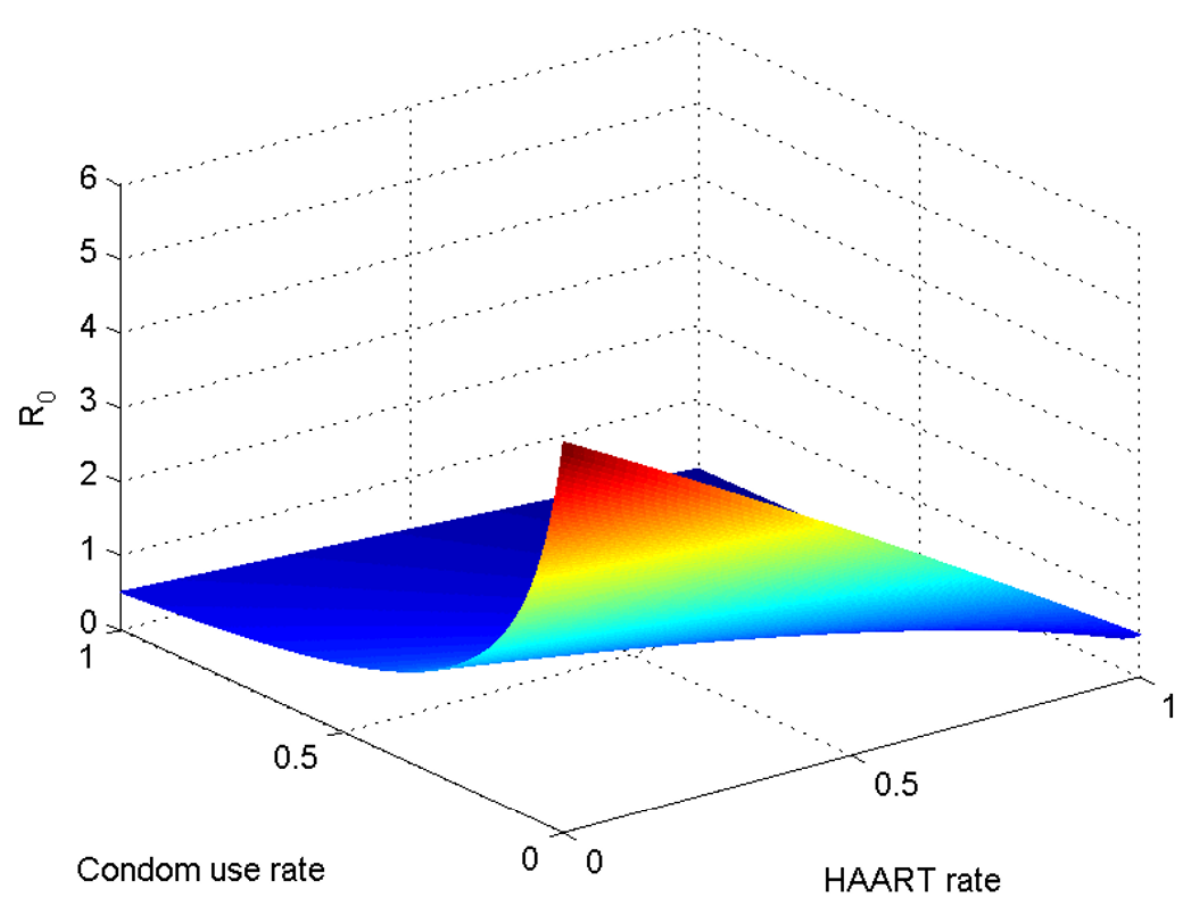

\section{Figure 5}

$R_{0}$ as a function of the HAART and condom rates. $R_{0}$ as a function of the HAART and condom rates. All other parameters were the same as in Figure 4. This figure illustrates the feasibility of controlling the HIV epidemic within the MSM population via a combination of condom use and HAART program.

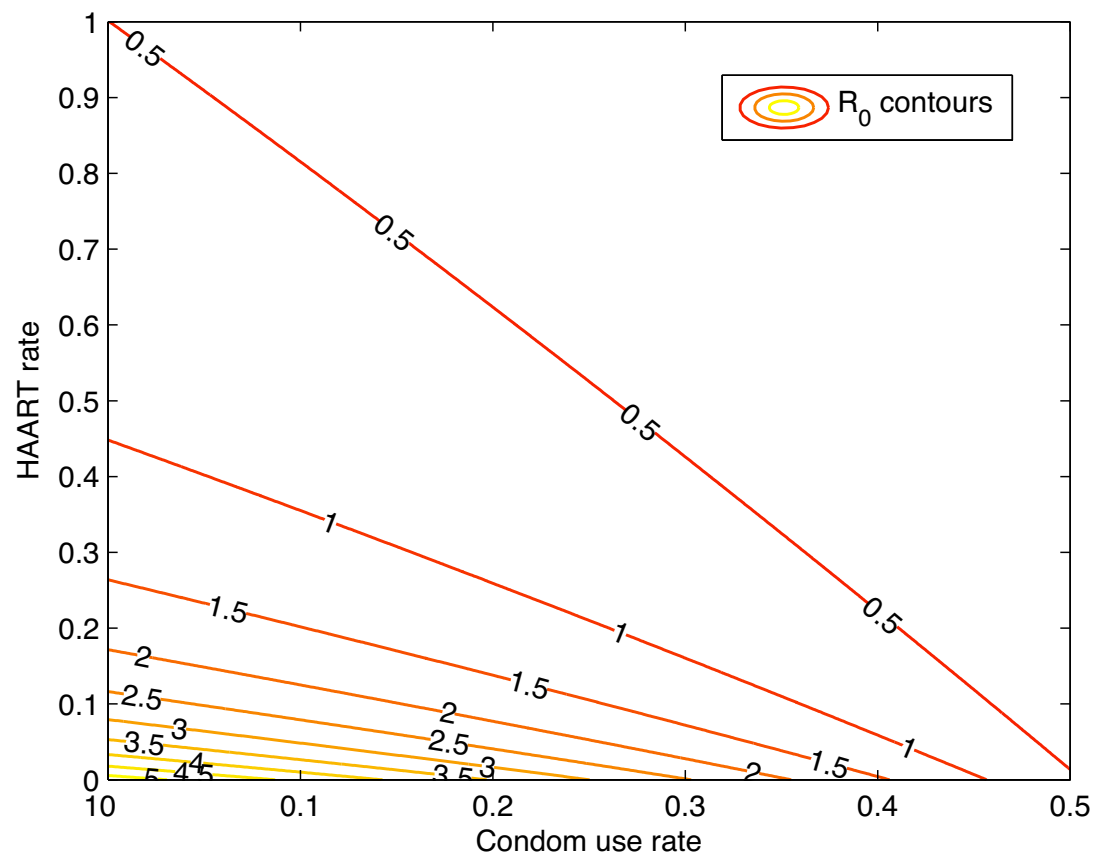

Figure 6

Contour plots of $R_{0}$ as HAART and condom rates vary. Contour plots of $R_{0}$ as HAART and condom rates vary.

disinhibition is considered. Our simulations also suggest 


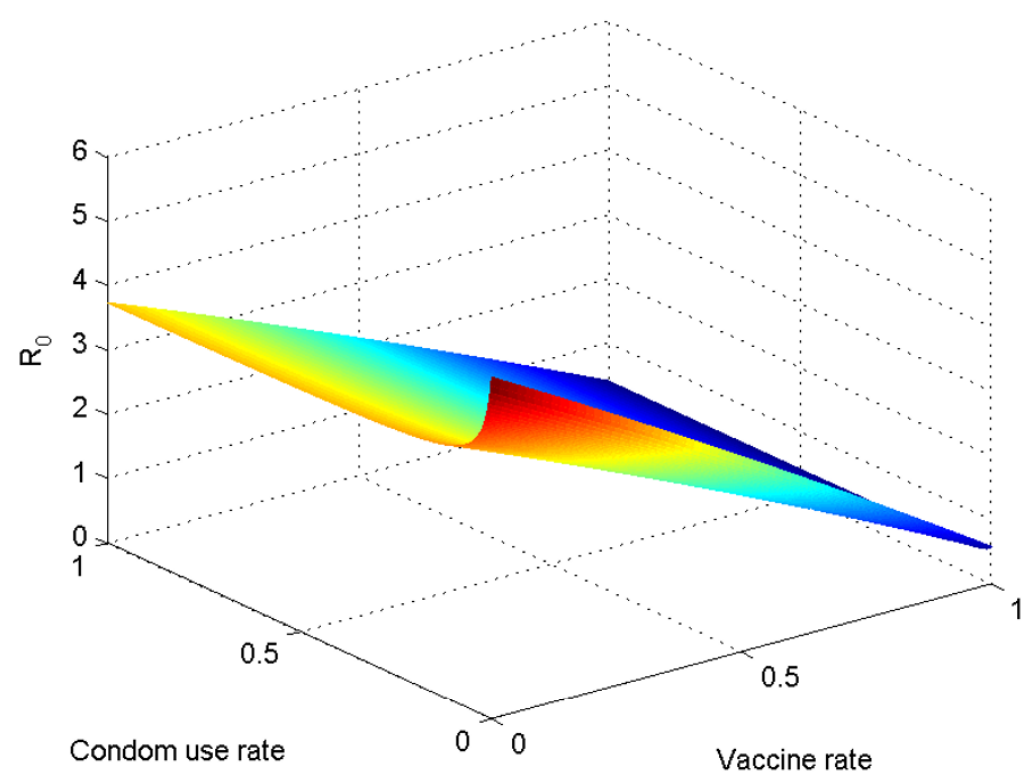

Figure 7

$R_{0}$ as a function of the vaccine and condom rates. $R_{0}$ as a function of the vaccine and condom rates. This figure illustrates the feasibility of controlling the HIV epidemic within the MSM population via a combination of condom use and vaccine program.

that having protected sexual behaviour has limited effect on controlling an epidemic in the MSM population, and medicine which can reduce the transmission and extend the lifespan of the infected has a complex impact.
There are three points that we should pay attention to. First, we suppose that most of these professional money boys are being in the Bottom Only category. This is from the investigation of China's current situation. Maybe there are some of them being in the other two categories. But

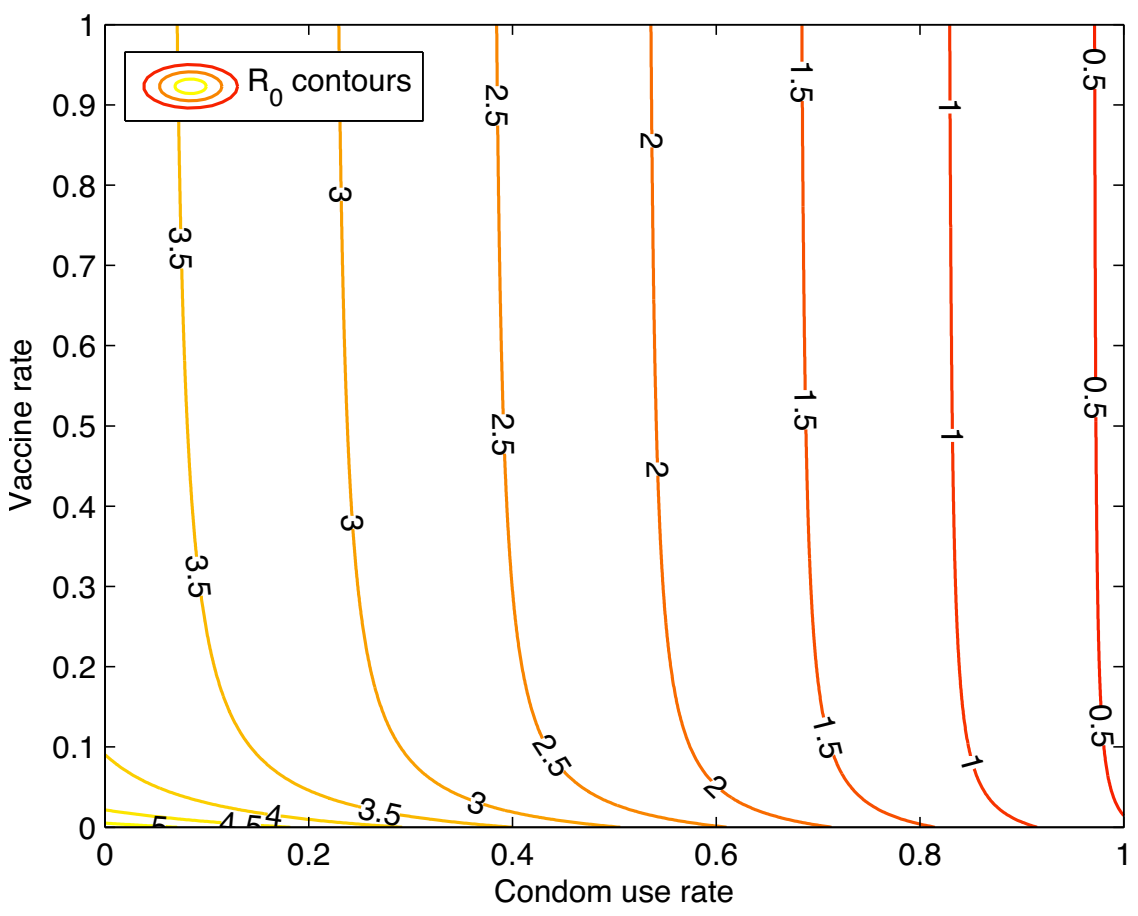

Figure 8

Contour plots of $\boldsymbol{R}_{\mathbf{0}}$ as vaccine and condom rates vary. Contour plots of $R_{0}$ as vaccine and condom rates vary. 


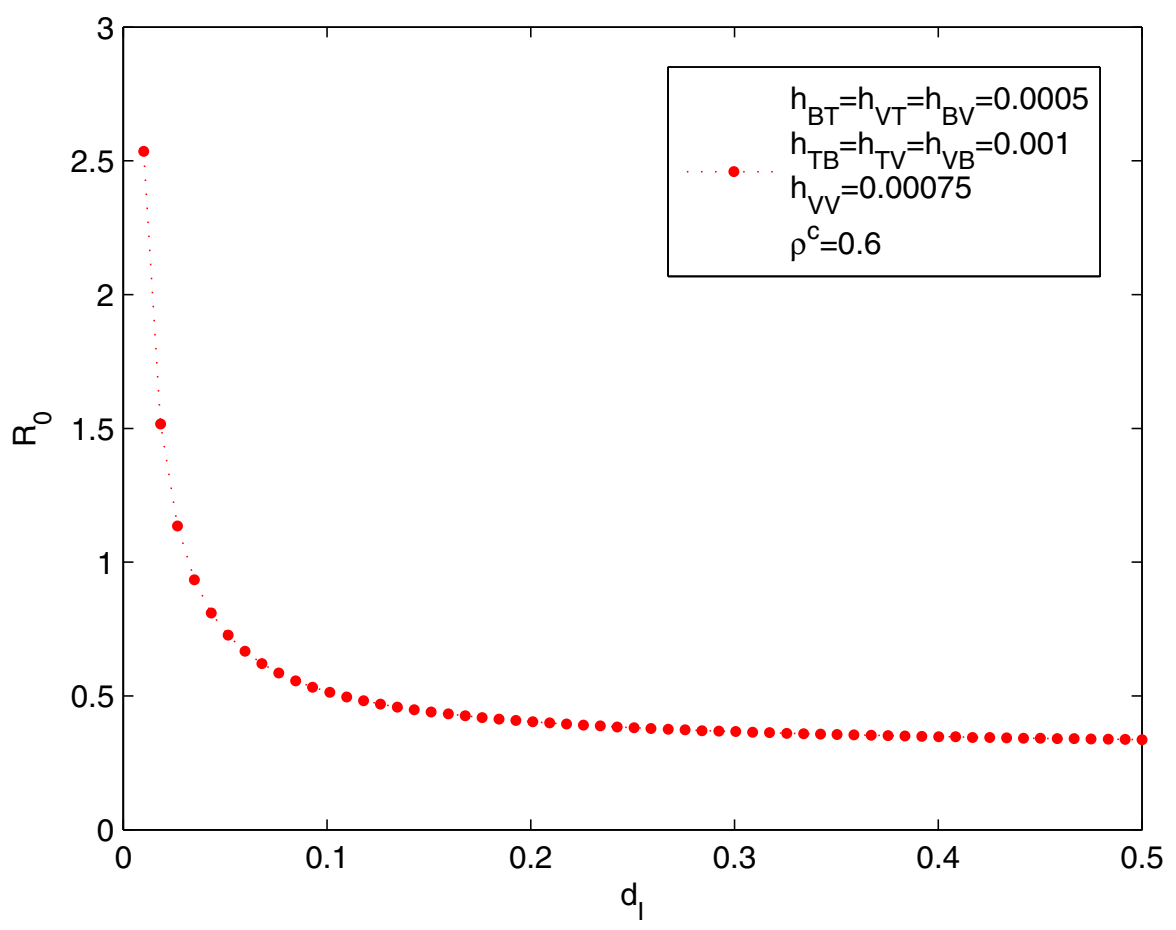

\section{Figure 9}

The relation between $\boldsymbol{R}_{\mathbf{0}}$ and $\boldsymbol{d}_{\boldsymbol{l}}$ follows a power law. The relation between $R_{0}$ and $d_{l}$, power law. Parameters used were as in Case 3 of Table 2. $R_{0}$ is a decreasing function of the death rate $d_{1}$, following a power law at least asymptotically.

since the proportions are small, the effect should be also very limit to the final outcomes. Second, considering the large variation of the data of different sexual partners for each MSM, which is possible to obey a power-law distribution, maybe the complex network (such as the ScaleFree network) is a more suitable method to model the spreading of HIV in MSM. This is also what we want to try in our next work. Third, many MSM in China, whether occasionally or frequently having sex with men, do not necessarily regard themselves as homosexual or bisexual. They are very often married. Even if they are not, they may have sex with women as well. This applies particularly to those societies wherein marriage is strongly promoted by the society and the family. This is largely true for rural workers, most of whom are married. Thus, as a bridging population, infected MSM transmit the infection to their heterosexual partners and thereafter to the general community.

\section{Competing interests}

The authors declare that they have no competing interests.

\section{Authors' contributions}

JL and JW are responsible for the model building and theory analysis. LC is responsible for simulations. YR conducted data collection and analysis. YS is responsible for designing the study, identifying the key indicators, and providing scientific discussion and application of them to the epidemic and public health efforts.

\section{Additional material}

\section{Additional file 1}

Click here for file

[http://www.biomedcentral.com/content/supplementary/14712458-9-S1-S10-S1.pdf]

\section{Acknowledgements}

JL's work was supported in part by the Natural Science Foundations of China (Grant No.10701053 and No. 10531030), by the China National Grand Program on Key Infectious Disease Control (2008ZX1000I-002 project 2), and by the Shanghai Leading Academic Discipline Project (S30 104). JW's work was supported by the Canada Research Chairs Program, by the Natural Sciences and Engineering Research Council of Canada, and by the Mathematics for Information Technology and Complex Systems. This work was also supported by a CRC-IDRC International Research Chair Program: Canada-China Program on Disease Modeling and Management (1045 I9-0I8) and the Ministry of Science and Technology of China (2007DFC30230). 
This article has been published as part of BMC Public Health Volume 9 Supplement I, 2009: The OptAIDS project: towards global halting of HIVIAIDS. The full contents of the supplement are available online at http:// www.biomedcentral.com/ $|47|-2458 / 9$ ? issue $=$ SI .

\section{References}

I. MSM 19 times more likely to be infected with HIV, says AIDS group Fridae: Empowering Gay Asia 2008 [http://www.fridae.com/ newsfeatures/2008/08/05/2099.msm-19-times-morelikely-to-beinfected-with-hiv-says-aids-group].

2. HIV infection rate climbing among gay men [http://www.chi nadaily.com.cn/china/2006-10/22/content 7/3884.htm]

3. $\mathrm{Li}$ Y: Selected Works of Li Yinhe. Neimenggu University Press; 2006.

4. Qu SQ, Zhang DP, Wu YH, Zhu H, Zheng XW: Seroprevalence of HIV and risk behaviours among men who have sex with men in a northeast city of China. Chin J STD/AIDS Prev Cont 2002, 8: $145-147$

5. Tong G: An Inquiry into Commercial Sex in the Community of Men Who Have Sex with Men in China. Beijing Gender Health Education Institute; 2007.

6. Tong G: MSM Volunteers Participation in HIVIAIDS Prevention Activities. Public Publication; $200 \mathrm{I}$.

7. Tong G: Research on the HIVIAIDS behavioural Intervention among MSM. Beijing Gender Health Education Institute; 2004.

8. Valle SD, Evangelista AM, Velasco MC, Kribs-Zaleta CM, Schmitz SFH: Effects of education, vaccination and treatment on HIV transmission in homosexuals with genetic heterogeneity. Mathematical Biosciences 2000, 187: I I I-I33.

9. Breban R, McGowan I, Topaz C, Schwartz EJ, Anton P, Blower S: Modeling the potential impact of rectal microbicides to reduce HIV transmission in bathhouses. Mathematical Biosciences and Engineering 2006, 3:450-466.

10. Tan WY, Xiang ZH: A state space model for the HIV epidemic in homosexual populations and some applications. Mathematical Biosciences 1998, 152:29-61.

II. Yee N: 2002 [http://www.nickyee.com/ponder/topbottom.html].

12. Hogg RS, Rhone SA, Yip B, Sherlock C, Conway B, Schechter MT, O'Shaughnessy MV, Montaner JS: Antiviral effect of double and triple drug combinations amongst HIV-infected adults: lessons from the implementation of viral load-driven antiretroviral therapy. AIDS 1998, 12:279-84.

13. Blower SM, Gershengorn HB, Grant RM: A tale of two futures: HIV and antiretroviral therapy in San Francisco. Science 2000, 287:650-654.

14. The total number of rural workers at the end of 2008 is 225,420,000 [http://www.cpirc.org.cn/tjsj/] tjsj cy detail.asp?id $=1047 \mathrm{I}]$

15. Diekmann O, Heesterbeek JAP, Metz JAJ: On the definition and the computation of the basic reproduction ratio $\mathrm{RO}$ in models for infectious diseases in heterogeneous populations. J Math Biol 1990, 28:s365.

16. High-risk behaviours and HIV/syphilis prevalence among men who have sex with men in Beijing. China CDC; 2008.

17. Abbas UL, Anderson RM, Mellors JW: Potential impact of antiretroviral therapy on HIV-I transmission and AIDS mortality in resource-limited settings. J Acquir Immune Defic Syndr 2006, 4I:632-4I.

18. Traditional Chinese medicine gives hope to HIV infected people [209794.html]

19. An anti-HIV traditional Chinese medicine has won state invention patent [http://zyb.gzst.gov.cn/dongtai.asp?id=506]

20. Porco TC, Martin JN, Page-Shafer KA, Cheng A, Charlebois E, Grant $\mathrm{RM}$, Osmond DH: Decline in HIV infectivity following the introduction of highly active antiretroviral therapy. AIDS 2004, I 8:8I-88.

21. Deschamps MM, Pape JW, Hafner A, Johnson WD Jr: Heterosexual transmission of HIV in Haiti. Annals of Internal Medicine 1996, I 25(4):324-330.
Publish with Biomed Central and every scientist can read your work free of charge

"BioMed Central will be the most significant development for disseminating the results of biomedical research in our lifetime. "

Sir Paul Nurse, Cancer Research UK

Your research papers will be:

- available free of charge to the entire biomedical community

- peer reviewed and published immediately upon acceptance

- cited in PubMed and archived on PubMed Central

- yours - you keep the copyright

Submit your manuscript here:

http://www.biomedcentral.com/info/publishing_adv.asp
BiolMedcentral 